\title{
Multiculturalidad y diversidad funcional en las cartas de servicio y sedes web de las bibliotecas públicas estatales y bibliotecas universitarias de España
}

\author{
Multiculturalism and functional diversity in the service charters and web sites \\ of the Spanish state public libraries and university libraries
}

\section{Laura Bravo-Fernández, Cristina Faba-PéRez}

Facultad de Ciencias de la Documentación y la Comunicación, Universidad de Extremadura. Plazuela Ibn Marwan, s/n, 06007 Badajoz (España), laurabravofe@gmail.com, cfabper@unex.es

\begin{abstract}
Resumen
Se estudia el nivel de implicación que tienen las 53 bibliotecas públicas estatales y las 76 bibliotecas universitarias de España con los colectivos multicultural y con diversidad funcional, utilizando como método el análisis detallado de sus cartas de servicio y de sus sedes web, con el fin de comprobar si se hace mención explícita a servicios y recursos especiales para los colectivos señalados y cómo se realiza dicha mención. Como resultado principal obtenemos que, tanto la multiculturalidad, como las personas con diversidad funcional, se encuentran muy poco representadas en las cartas de servicio de ambos tipos de bibliotecas, con porcentajes inferiores al $5 \%$ en multiculturalidad y al $35 \%$ en diversidad funcional, y si bien estos resultados resultan más elevados en las sedes web de las dos tipologías bibliotecarias, con porcentajes inferiores al $29 \%$ para el colectivo multicultural y al $45 \%$ en las personas con diversidad funcional, no podemos concluir favorablemente, ya que consideramos importante fomentar, desde el $100 \%$ de las bibliotecas públicas y universitarias, la sensibilización social acerca de los valores positivos de ambos colectivos.
\end{abstract}

Palabras clave: Multiculturalidad. Diversidad funcional. Cartas de servicio. Sedes web. Bibliotecas públicas estatales. Bibliotecas universitarias. España.

\section{Introducción}

Estamos siendo testigos de una sociedad cambiante que demanda la reestructuración de sus servicios e instituciones. Este cambio ha cuestionado la necesidad de las bibliotecas en un momento en el que el acceso a la información y a la documentación digital es cada vez más ágil y transparente a través de la Red. Este servicio público ha perdido la exclusiva de ser casi el único proveedor de información, y la llegada de los recursos digitales está provocando que menos ciudadanos visiten de manera presencial las bibliotecas (Horrigan, 2015, citado en Sánchez-García

\begin{abstract}
The level of involvement that the 53 state public libraries and the 76 university libraries of Spain have with their multicultural and functional diversity groups is analyzed. For that, their service charters and their web sites were checked for explicit mentions of special services and resources for the aforementioned groups. As a main result, we obtain that both multiculturalism and people with functional diversity are underrepresented in the service charters of both types of libraries, with percentages of less than $5 \%$ for multiculturalism and $35 \%$ for functional diversity, and although these results are higher in the web sites of both library types, with percentages of less than $29 \%$ for the multicultural community and $45 \%$ for people with functional diversity, we cannot conclude favorably, since we consider it important to promote social awareness about the positive values of multiculturalism and functional diversity from the $100 \%$ of public and university libraries.
\end{abstract}

Keywords: Multicultural. Functional diversity. Service charters. Web sites. State public libraries. University libraries. Spain.

y Yubero, 2016). En un momento de incertidumbre, la sostenibilidad de estas instituciones dependerá de su capacidad para mostrar su utilidad a los ciudadanos.

El abanico de servicios ofrecidos por las bibliotecas en general es muy variado (préstamo, información bibliográfica, dinamización, alfabetización informacional y digital, etc.). Sin embargo, servicios y/o recursos para ciertos colectivos, como el multicultural y con diversidad funcional, parece que no se hallan suficientemente representados en las bibliotecas. Por eso, con este trabajo queremos comprobar el nivel de implicación 
que tienen las bibliotecas públicas estatales y las bibliotecas universitarias de España con los dos colectivos mencionados, examinando si en sus cartas de servicio y en sus sedes web se hace mención explícita a servicios y/o recursos especiales para ellos. Antes de continuar, queremos definir el sentido de las diversas variables que intervienen en este trabajo:

Multiculturalidad. La IFLA (International Federation of Library Associations and Institutions) define la multiculturalidad en el Manifiesto IFLA por la biblioteca multicultural (2008), basándose en la Declaración Universal de la UNESCO sobre la Diversidad Cultural de 2001, como:

La «diversidad cultural» o «multiculturalidad» se refieren a la coexistencia e interacción armónica de culturas diferentes, donde «la cultura debe ser considerada como el conjunto de los rasgos distintivos espirituales y materiales, intelectuales y afectivos que caracterizan a una sociedad o a un grupo social y que abarca, además de las artes y las letras, los modos de vida, las maneras de vivir juntos, los sistemas de valores, las tradiciones y las creencias». La diversidad cultural o la multiculturalidad son la base de nuestra fuerza colectiva, tanto en nuestras comunidades locales como en nuestra sociedad global.

Además, en las pautas de la IFLA tituladas Comunidades multiculturales: directrices para el servicio bibliotecario (2009), algunos grupos son descritos como "tradicionalmente desatendidos" en el seno de una sociedad multicultural, en concreto, minorías inmigrantes, personas que buscan asilo o trabajadores migratorios.

Diversidad funcional. Según la Organización Mundial de la Salud (OMS):

Discapacidad es un término general que abarca las deficiencias, las limitaciones de la actividad y las restricciones de la participación. Las deficiencias son problemas que afectan a una estructura o función corporal; las limitaciones de la actividad son dificultades para ejecutar acciones o tareas, y las restricciones de la participación son problemas para participar en situaciones vitales. Por consiguiente, la discapacidad es un fenómeno complejo que refleja una interacción entre las características del organismo humano y las características de la sociedad en la que vive.

La discapacidad puede ser intelectual, física, sensorial o psíquica y, desde hace unos años, el término "discapacidad" se ha sustituido por "diversidad funcional", que según Rodríguez Díaz y Ferreira (2010) ha sido reclamado por el propio colectivo de personas con discapacidad, por ello, será el término que adoptaremos en el presente trabajo:

La diversidad funcional [...] conceptualiza el fenómeno en términos de una singularidad funcional, de desenvolvimiento, apunta a la riqueza de un universo social de lo humano crecientemente caracterizado por la diversidad. En lugar de partir de una insuficiencia, lo hace, podríamos decir, de una "originalidad", potencialmente, entonces, creativa y enriquecedora. Y lo que reclama es un cambio de óptica que la tome en consideración en lugar de asumirla como algo negativo. La principal virtud de este tercer discurso es que es fruto de una decisión "autónoma" del colectivo de personas con discapacidad que rechaza las imposiciones nominales y quiere reconocerse por lo que es y no por aquello de lo que supuestamente carece.

Cartas de servicio. Es un instrumento de gestión mediante el cual las organizaciones públicas informan a los ciudadanos sobre los servicios que ofrecen, difundiendo sus compromisos de calidad y los derechos de los usuarios. Su objetivo principal es ayudar a mejorar los servicios que ofrecen las organizaciones públicas (Candia y Maderni, 2011). Tienen como finalidad: garantizar el derecho de los ciudadanos a la información; comunicar su compromiso de servicio y calidad; estimular toda iniciativa de mejora del servicio; responder y anticiparse a las demandas ciudadanas; y divulgar las prestaciones y servicios del centro. En definitiva, según González: (2014):

Constituye una nueva herramienta de difusión y compromiso con los ciudadanos, que, a diferencia de las simples guías, incorporan unos sistemas de medición del rendimiento, a través de indicadores, para comprobar el grado de cumplimiento de esos objetivos de manera segura, definiendo por último la calidad del servicio que se presta a los ciudadanos.

Sede web. Algunos investigadores como Aguillo (1998) han definido una sede web como:

Conjunto de páginas ligadas jerárquicamente, que constituyen una unidad diferenciada e independiente, tanto en función de contenidos (unidad documental), como de organización responsable (unidad institucional). Debe tener identidad y ser reconocible.

Biblioteca pública. La IFLA define la biblioteca pública en sus Directrices para el Desarrollo del Servicio de Bibliotecas Públicas (2001), como:

Una organización establecida, respaldada y financiada por la comunidad, ya sea por conducto de una autoridad u órgano local, regional o nacional, o mediante cualquier otra forma de organización colectiva. Brinda acceso al conocimiento, la información y las obras de la imaginación gracias a toda una serie de recursos y servicio y está a disposición de todos los miembros de la comunidad por igual, sean cuales fueran su raza, nacionalidad, edad, sexo, religión, idioma, discapacidad, condición económica y laboral y nivel de instrucción.

Biblioteca universitaria. En 2013, la XXI Asamblea Anual de REBIUN (Red de Bibliotecas Universitarias Españolas), aprobó la nueva definición del modelo de biblioteca del siglo XXI: 
Un centro de recursos para el aprendizaje, la docencia y la investigación y las actividades relacionadas con el funcionamiento y la gestión de la Universidad/Institución en su conjunto. La biblioteca tiene como misión facilitar el acceso y la difusión de los recursos de información y colaborar en los procesos de creación del conocimiento a fin de contribuir a la consecución de los objetivos de la Universidad/Institución, realizando las actividades que esto implica de forma sostenible y socialmente responsable.

La relación entre los conceptos mencionados es evidente desde el momento en que las bibliotecas públicas y las universitarias, ya sea en sus instalaciones físicas y/o en sus sedes web, deben suministrar servicios a los ciudadanos bajo un prisma de responsabilidad social, lo que implica tener en cuenta a los colectivos multiculturales y con diversidad funcional. Además, estas instituciones deberían disponer de cartas de servicio donde explicitaran su relación con dichos colectivos a fin de cumplir uno de los objetivos más importantes de las cartas de servicio: "garantizar el derecho de todos los ciudadanos a la información".

\subsection{Bibliotecas públicas y multiculturalidad}

La sostenibilidad de las bibliotecas en general y de las bibliotecas públicas en particular, dependerá de su capacidad para mostrar su utilidad a los ciudadanos, a sus administradores y a los políticos responsables de asignarle presupuesto (Varela-Prado y Baiget, 2012, citado en Sánchez-García y Yubero, 2016). Dicha utilidad se ve reflejada, sin duda, en la realidad multicultural en la que se encuentra inmersa la sociedad actual, siendo la biblioteca pública un elemento de cohesión e integración de todas las culturas. En una sociedad cada vez más heterogénea, la tasa de migración internacional ha contribuido a la diversidad cultural en muchas naciones, por ello, "las bibliotecas deben luchar por fondos, espacio y personal capacitado para atender las necesidades multilingües y multiculturales de sus comunidades de manera efectiva" (Abraham, 1986).

El surgimiento del compromiso social de la biblioteca viene marcado por la promulgación en 1948 de la Declaración Universal de los Derechos Humanos de las Naciones Unidas. Con esta Declaración, la biblioteca avanza y ayuda a difundir su responsabilidad hacia el compromiso social, lo que fomenta que muchos bibliotecarios colaboraren en el desarrollo de servicios bibliotecarios más justos, equitativos y solidarios (Magán y Gimeno, 2008).

Las directrices internacionales sobre bibliotecas públicas también han reflejado esta realidad social y, poco a poco, han ido haciendo referencia a las minorías étnicas, lingüísticas y culturales en su normativa, hasta establecer unas normas específicas sobre las comunidades multiculturales (García y Monje, 2002). Así, las bibliotecas llamadas multiculturales, multiétnicas o interculturales se mencionan ya desde la primera versión del Manifiesto de las Bibliotecas Públicas de la IFLA en 1949, que señala la apertura de dichas instituciones a todos los miembros de la comunidad, independientemente de su ocupación, creencias religiosas o afiliación política, clase o raza, hasta el Manifiesto de la Biblioteca Multicultural de IFLA (2008), que indica:

Cada individuo tiene derecho a una gama completa de servicios de biblioteca e información, y las bibliotecas deben servir a todos los miembros de la comunidad sin discriminación, independientemente de su edad, nacionalidad, religión u orientación política, y satisfacer las solicitudes de los usuarios utilizando tecnología moderna si es necesario.

Las bibliotecas públicas son agentes del cambio social y grandes herramientas para la integración de los inmigrantes. Son espacios donde los usuarios pueden acceder fácilmente a la información, no solo para aumentar sus conocimientos, sino también para mejorar sus habilidades para participar en la sociedad. Los inmigrantes, refugiados y residentes extranjeros deben desarrollar habilidades de alfabetización informativa para que puedan integrarse en su país de adopción. Saber cómo acceder, usar y comunicar información de manera efectiva permitirá la inclusión social de los recién llegados. Por lo tanto, desarrollar programas especiales de alfabetización informacional para equipar a dichos grupos con habilidades de alfabetización informativa debe estar entre las prioridades de las bibliotecas públicas (Oğuz y Kurbanoğlu, 2013). Este colectivo valora positivamente la socialización que logran en la biblioteca como espacio confortable e inclusivo en el que se sienten integrados y donde se desenvuelven sin limitaciones para fines lúdicos o funcionales (Gómez-Hernández, Hernández-Pedreño y Romero-Sánchez, 2017). De hecho, los nuevos inmigrantes menores de edad buscan en ella apoyo escolar y aprender el idioma, lo que supone para la biblioteca un nuevo reto para ayudarles a integrarse en la sociedad.

El personal de las bibliotecas debe promover acciones que tiendan a reducir las desigualdades sociales, favoreciendo el acceso de todos los ciudadanos a la cultura, la información y la formación, siendo especialmente sensibles con aquellos colectivos más vulnerables. La complejidad de lo social hace necesaria la incorporación en las plantillas de especialistas formados en los procesos y prácticas socioeducativas, así como la intervención con colectivos con necesidades especiales (Sánchez-García y Yubero, 2016). 
Con respecto a determinadas investigaciones sobre la relación entre las bibliotecas públicas y la multiculturalidad, en el caso de EEUU, nos encontramos trabajos como el de Novotny (2003), Wang (2012) o Thomas, et al. (2016), que muestran cómo las comunidades de todo el país luchan por integrar a los recién llegados en las esferas económica, cultural o política de la sociedad estadounidense. En España, mención especial merece la creación en 2014 de la Fundación Biblioteca Social, una asociación sin ánimo de lucro, que tiene como misión realizar proyectos en diversas comunidades autónomas para visibilizar la importancia de las bibliotecas públicas a la hora de ayudar a compensar las desigualdades sociales. Muy interesantes también son los trabajos de CervantesMartínez y Navas-Luque (2013) y Cervantes-Martínez, Navas-Luque y Cuadrado-Guirado (2015, 2019) sobre los servicios a inmigrantes en las bibliotecas públicas de Barcelona y Almería.

\subsection{Bibliotecas universitarias y multiculturalidad}

A fines del XIX, la biblioteca de carácter público presta un importante apoyo a la educación básica y también universitaria. A partir de 1850, países europeos como Alemania, Francia o Gran Bretaña llevan a cabo políticas educativas que dan lugar a la creación de escuelas y bibliotecas. EI afianzamiento de las universidades a lo largo del siglo XIX y la creación de nuevos centros universitarios en Europa y Estados Unidos, conlleva un mayor auge y compromiso social de las bibliotecas universitarias. Tradicionalmente, dicho compromiso social se ha relacionado con actividades de extensión bibliotecaria como exposiciones, presentaciones de libros, campañas de marketing y publicidad, etc. En muchas ocasiones, esta responsabilidad se sale de las actividades propias de un bibliotecario, por lo que es común considerar las acciones dirigidas a la responsabilidad social como tareas impropias de la profesión, más centradas en aspectos técnicos.

Sin embargo, la biblioteca universitaria tiene una responsabilidad social que no puede eludir y debe acometer desarrollando, entre otras actividades sociales, programas multiculturales. Un campus multicultural solo puede surgir con la creación de un entorno que brinde el apoyo y el estímulo que permita el crecimiento académico y el éxito de los estudiantes internacionales. Los bibliotecarios cumplen una función especial como provisores de información desempeñando un papel importante para garantizar el éxito académico de los estudiantes universitarios. Para lograrlo, en primer lugar, deben aumentar su comprensión básica del impacto cultural y económico de los estudiantes internacionales a nivel nacional y universitario (Kumar y Suresh, 2001). Una de las acciones para garantizar el éxito de los estudiantes de diversas culturas es incluir materiales multiculturales/multilingües en colecciones de bibliotecas académicas para reflejar la herencia de estudiantes nativos e internacionales. Agregar estos materiales ayuda a crear una atmósfera acogedora para todas las poblaciones, al tiempo que proporciona materiales para contrarrestar los prejuicios y aumentar la base de conocimientos y las competencias comunicativas de aquellos que no están acostumbrados a entornos multiculturales (Schomberg y Grace, 2005).

Investigaciones sobre las bibliotecas universitarias y la multiculturalidad en EEUU muestran cómo la integración de la diversidad cultural en las universidades mejoraría si fuera promovido desde los planes de estudio de las titulaciones en Biblioteconomía y Documentación (Library and Information Science, LIS (Kumasi y Manlove, 2015) o cómo el hecho de incentivar la comunicación sobre la diversidad y la inclusión social entre los estudiantes, el profesorado y la biblioteca universitaria un clima positivo en el campus (Everett, 2018). Como señalan Magán y Gimeno (2008), en España, muchas universidades han propuesto planes en sus bibliotecas para la inclusión de la multiculturalidad en las mismas (Universidad Politécnica de Cataluña, Universidad de Murcia, o Universidad Complutense de Madrid, entre otras).

\subsection{Bibliotecas públicas y diversidad funcional}

Las bibliotecas no han conseguido abordar del todo una oferta de servicios para los usuarios con diversidad funcional, esto se debe a que dichos servicios se encuentran limitados a la atención de algunas discapacidades. $Y$ es por eso que los bibliotecarios se plantean cómo resolver esta situación, tomando las medidas necesarias que ayuden a los usuarios con diversidad funcional a tener contacto con toda la información. El cambio debe estar enfocado en el acceso a los edificios de las bibliotecas, y también en las necesidades del público al que se dirige en lo referente al mobiliario, al equipo y la colección. Además, es necesaria la preparación del personal bibliotecario para comprender el tipo de necesidades que este grupo de usuarios necesita (Santillán-Rivero y Valles-Valenzuela, 2004).

Todas las bibliotecas deberían contar con las condiciones mínimas que le permitan el cumplimiento de sus funciones. Sin embargo, las necesidades de las personas con diversidad funcional están muy poco consideradas. Los derechos de los discapacitados establecen que las Naciones deberían proteger los derechos de estos ciudadanos a través de disposiciones constitucionales, que afirmen la dignidad de las personas y su 
derecho a tratamiento y acceso a los servicios, sin importar el desarrollo económico del país. Además, las personas discapacitadas deben tener los mismos derechos que el resto de los ciudadanos y no se les puede privar de ellos (SantiIlán-Rivero y Valles-Valenzuela, 2004).

Con respecto a investigaciones sobre la relación entre las bibliotecas públicas y la diversidad funcional, en EEUU podemos señalar que determinadas leyes, como la Ley de Estadounidenses con Discapacidades de 1990 (que hacía hincapié en el acceso a la colección de la biblioteca para personas con discapacidad) ayudaron considerablemente en la inclusión de este colectivo en las bibliotecas públicas (Khailova, 2006). También se han investigado, por ejemplo, programas inclusivos en las bibliotecas públicas para niños con dificultades auditivas (Bushman y Fagan, 2019). En el caso de España, las bibliotecas públicas han propuesto diferentes medidas, como la desarrollada hace ya unos años por la Comunidad de Madrid con actividades tales como talleres de iniciación al lenguaje de signos para personas con discapacidad auditiva (Moral, Pedreira y Sanz, 2008).

\subsection{Bibliotecas universitarias y diversidad funcional}

El actual sistema universitario está cambiando, siendo fundamental la adecuación de sus servicios a todos los miembros universitarios, independientemente de sus capacidades. Small, Myhill, y Herring-Harrington, L. (2015) consideran elemental incrementar la conciencia de los bibliotecarios sobre la importancia de proporcionar programas y servicios efectivos, bien diseñados e inclusivos a los usuarios con diversidad funcional, garantizando que todas las bibliotecas sean física y virtualmente accesibles para todos. La Agenda 2030 de los Objetivos de Desarrollo Sostenible contempla un mundo de respeto universal por los derechos y la dignidad humanos, la igualdad y la no discriminación, un mundo equitativo, tolerante, abierto y socialmente inclusivo en el que las necesidades de los más vulnerables sean cumplidos y derechos inclusivos como la educación, el acceso a los servicios (incluida la información) y el empleo para personas con discapacidad (Majinge y Mutula, 2018).

La protección de los derechos básicos de las personas con diversidad funcional, la eliminación de la discriminación social y la reducción de la brecha de accesibilidad son parte integral de la inclusión social universitaria (Majinge y Mutula, 2018). Sin embargo, aún hoy en día, muchas bibliotecas universitarias de todo el mundo carecen de la capacidad de ofrecer un servicio de información efectivo para las personas, por ejemplo, con discapacidad visual. En esta línea, la escasez de recursos de información en braille y en letra grande, los edificios de biblioteca inaccesibles y la falta de tecnologías de asistencia son los problemas que afectan al acceso y el uso de la información por parte de los estudiantes con discapacidad visual. Por ello, donde no existan políticas y actuaciones adecuadas deben proclamarse para que las tecnologías de asistencia, la infraestructura y los recursos estén disponibles para suavizar estos desafíos a los que se enfrentan estos estudiantes en universidades como en las de Sudáfrica (Mutula y Majinge, 2016).

Algunas investigaciones sobre las bibliotecas universitarias y la diversidad funcional en EEUU se centran en los beneficios de la accesibilidad web para este colectivo, de ahí su compromiso en cumplir con las Pautas de Accesibilidad al Contenido en la Web (Lush, 2015). También ahondan en los beneficios que supone la inclusión en los estudios de Biblioteconomía y Documentación (Library and Information Science, LIS) de contenidos sobre accesibilidad web (Green y Huprich, 2009) y diversidad funcional (Mulliken y Djenno, 2017). En España, en los años noventa, el entorno universitario comienza a acercarse a los estudiantes con diversidad funcional con la puesta en marcha de servicios y programas adaptados, conocidos como servicios de apoyo, unidades de integración, etc., (Millán Reyes, 2010). Esto ha llevado a las universidades españolas a establecer como norma la igualdad reconociendo los derechos para los alumnos con discapacidad (Serna Bilbao, 2014).

\subsection{Objetivos}

El objetivo principal de este trabajo consiste en comprobar si las bibliotecas públicas estatales y las bibliotecas universitarias (públicas y privadas) de España representan adecuadamente al colectivo multicultural y/o con diversidad funcional, teniendo en cuenta si en sus cartas de servicio y en sus sedes web, ofertan servicios y/o recursos destinados a ellos.

\section{Metodología}

\subsection{Datos}

Para seleccionar el listado de bibliotecas públicas del Estado recurrimos al Directorio del Ministerio de Cultura y Deporte (1), recopilando un total de 53 bibliotecas. Y para obtener las bibliotecas universitarias de España (públicas y privadas) consultamos el Listado ofrecido por la Conferencia de Rectores de las Universidades Españolas (CRUE) (2), reuniendo un total de 76 bibliotecas centrales universitarias. Como se observa, 
en ambos casos, utilizamos fuentes de carácter oficial para recopilar las bibliotecas y sus URL, que ascienden a 129.

\subsection{Metodología}

Con el fin de descubrir si las bibliotecas mencionadas con anterioridad recogen en sus cartas de servicio y en sus sedes web servicios y/o recursos para el colectivo multicultural y con diversidad funcional, seguimos el procedimiento siguiente:

1. Para la recogida de información se han creado dos plantillas en Excel, una para las bibliotecas públicas estatales y otra para las bibliotecas universitarias. Las columnas de dichas plantiIlas son: 'Código de la biblioteca', 'Denominación', 'Titularidad' (solo en el caso de las universitarias, para distinguir entre pública o privada), 'Provincia', portal web 'URL', 'Cartas de servicio' y 'Sede web', A su vez, 'Cartas de servicio' y 'Sede web', se subdividen en: 'Ubicación de la carta de servicios', 'Servicios para colectivos multiculturales' (Dónde y Cómo se recogen) y 'Servicios para colectivos con diversidad funcional' (Dónde y Cómo se recogen). En el caso de que se ofrezcan servicios para dichos colectivos se pondera siempre con ' 1 ' (independientemente de la cantidad de servicios o recursos de los que disponga) y con ' 0 ' en el caso de no poder localizar ninguno.

2. Con respecto a las 'Cartas de servicio', para conocer con exactitud el porcentaje de bibliotecas que sí disponen de ella en su sede web y las que no, llevamos a cabo un estudio pormenorizado que comenzó con la localización de la carta de servicios en la web de cada biblioteca por parte de los analistas, continuó con la consulta a través de los formularios disponibles en la web, y finalizó con llamadas telefónicas a los responsables de las bibliotecas. Con esta metodología certificamos que el $100 \%$ de los datos hallados son totalmente fiables. Una vez comprobada la disponibilidad de cartas de servicio, se rellena la columna 'Ubicación', con la información detallada sobre su localización en la web de la biblioteca.

3. A continuación, se comprueba si en dichas cartas se hace referencia a servicios y/o recursos para el colectivo multicultural y con diversidad funcional, y en caso positivo, se reIlenan las columnas 'Dónde', que hace referencia a la sección de la carta de servicios donde se halla dicha información, y 'Cómo' que señala la manera en la que se refleja la información sobre dichos colectivos.

4. El mismo procedimiento se sigue para las sedes web, observando si en ellas se hace referencia a servicios y/o recursos para el colectivo multicultural y con diversidad funcional y rellenando las columnas 'Dónde' y 'Cómo'.

5. Una vez recopilada toda la información, proceso llevado a cabo entre septiembre de 2019 y enero de 2020, procedemos a su análisis en los Resultados del trabajo.

\section{Resultados}

\subsection{Análisis de las cartas de servicio}

\subsubsection{Análisis general}

En primer lugar, tras hacer un estudio exhaustivo de las sedes web de las bibliotecas públicas estatales y bibliotecas universitarias de toda España para descubrir si disponen de cartas de servicio, se ha comprobado que 23 de las 53 bibliotecas públicas, sí disponen de cartas de servicio (menos de la mitad, $43,40 \%$ ) (Apéndice 1) frente a 30 bibliotecas (56,60\%) que no tienen (es preciso matizar que bibliotecas, como Granada o Murcia, han sido descartadas puesto que remiten a la carta de servicios de la Biblioteca Regional de la Comunidad Autónoma respectiva). Si lo comparamos con las 76 bibliotecas universitarias, existe una diferencia significativa, ya que 48 de ellas $(63,16 \%)$ cuenta con cartas de servicio y $28(36,84 \%)$ no disponen de ella (Figura 1$)$.

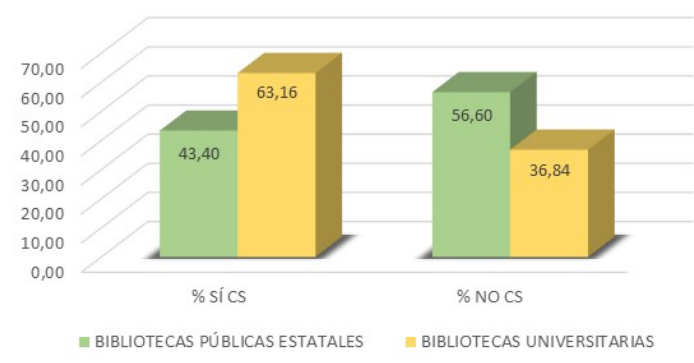

Figura 1. Cartas de servicios en webs de bibliotecas públicas estatales y universitarias: España

\subsubsection{Análisis específico por tipo de biblioteca}

\section{Bibliotecas públicas estatales}

Tras aplicar la metodología descrita, observamos que de las 23 bibliotecas que sí disponen de cartas de servicio, el $100 \%$ de las mismas (23 bibliotecas) han sido claramente localizadas por los analistas, lo que puede indicar una adecuada ubicación en sus sedes web, y de las 30 bibliotecas que no tienen cartas de servicio, el 16,67\% de ellas ( 5 bibliotecas) han sido ratificadas por la respuesta dada en el formulario disponible en la web, y el $83,33 \%$ (25 bibliotecas) por la respuesta telefónica de los bibliotecarios. 
Se ha realizado un análisis cualitativo detallado para mostrar si las cartas de servicio de las $23 \mathrm{bi}$ bliotecas públicas del Estado están correctamente ubicadas en las sedes web o, por el contrario, es complicada su localización. De esta manera, se han identificado cinco apartados generales en los que se hallan ubicadas las cartas de servicio: "Biblioteca", "Conócenos", "Información", "Normativa" y "Servicios". Estos apartados se encuentran, en su mayoría, en la página principal de las sedes web de las bibliotecas públicas, y su denominación invita a pensar que en ellos se puedan encontrar las cartas de servicio, por ello, se puede decir que las sedes web de las bibliotecas públicas del Estado con cartas de servicio las tienen accesibles. Hay que aclarar que algunas cartas de servicio dependen directamente de la categoría mencionada (por ejemplo, ConócenosCartas de Servicio; La Biblioteca-Cartas de Servicio; Información-Cartas de Servicio), pero otras no (por ejemplo, Conócenos-Biblioteca-Cartas de Servicio, o Información-Biblioteca-Cartas de Servicio). Excepto "Normativa" que siempre está incluida en otra. En estos últimos casos, se ha optado por incluir la biblioteca pública en el apartado cuyo nombre aparece en primer lugar.

El apartado en el que se han encontrado más cartas de servicio es "Conócenos", con 9 de las 23 bibliotecas con cartas de servicio $(39,13 \%)$, le sigue "Información" con 6 bibliotecas $(26,08$ $\%)$, "Biblioteca" con $4(17,39 \%)$ y, por último, con 2 bibliotecas $(8,69 \%)$ se encuentran los apartados de "Normativa" y "Servicios".

\section{Bibliotecas universitarias}

En este caso se observa que de las 48 bibliotecas que sí disponen de cartas de servicio (Apéndice 2), el 95,83\% de las mismas (46 bibliotecas) han sido claramente localizadas por los analistas, lo que puede significar que están relativamente bien ubicadas en sus sedes web, pero el $4,17 \%$ ( 2 bibliotecas) han debido ser confirmadas mediante formulario. De las 28 bibliotecas sin cartas de servicio, la mayoría de ellas, el 60,71\% (17 bibliotecas) han sido ratificadas por formulario y el 39,29 \% (11 bibliotecas) por la respuesta telefónica de los bibliotecarios.

Siguiendo el mismo procedimiento que en las bibliotecas públicas del Estado, en el estudio cualitativo de las 48 bibliotecas universitarias con cartas de servicio se han identificado los mismos cinco apartados generales en los que se ubican dichas cartas: "Biblioteca", "Conócenos", "Información", "Normativa" y "Servicios". Al igual que en las bibliotecas públicas, estos apartados se encuentran, en su mayoría, en la página principal de las sedes web de las bibliotecas universitarias, por lo que se puede decir que las sedes web de las bibliotecas universitarias con cartas de servicio las tienen accesibles. También en este caso, algunas cartas de servicio dependen directamente del apartado mencionado, pero otras no, en cuyo caso se ha optado por incluir la biblioteca en el apartado cuyo nombre aparece en primer lugar (tenemos que exceptuar el apartado "Normativa", que siempre depende de otro).

El apartado con un mayor número de cartas de servicio es "Biblioteca" y "Conócenos", donde se agrupan 11 bibliotecas en cada apartado $(22,92$ $\%)$, en "Normativa" y "Servicios", 10 bibliotecas en cada categoría (20,83 \%) y en "Información", $6(12,50 \%)$.

\subsubsection{Análisis de la multiculturalidad y la diversidad funcional en las cartas de servicio}

Este análisis se realiza teniendo en cuenta únicamente las 23 bibliotecas públicas del Estado y las 48 bibliotecas universitarias que actualmente sí disponen de cartas de servicio. Se quiere comprobar si en ellas se hace referencia (principalmente, mediante servicios o recursos específicos) a la multiculturalidad y a las personas con diversidad funcional, ya que, uno de los objetivos de toda carta de servicio es comunicar su compromiso de servicio y calidad con todos los ciudadanos. En este caso, el escrutinio de las cartas de servicio ha sido realizado exclusivamente por los analistas.

De manera general, se puede decir que el número de bibliotecas de ambas tipologías en cuyas cartas de servicio se hace referencia a los dos colectivos objeto de estudio es bastante escaso, especialmente para el colectivo multicultural con porcentajes muy bajos $(4,17 \%$ en bibliotecas universitarias, 2 bibliotecas de 48), o incluso nulo en las bibliotecas públicas estatales. Sin embargo, y aunque no sea mucho mayor la diferencia entre las dos tipologías de bibliotecas, un $34,78 \%$ de las bibliotecas públicas del Estado (8 bibliotecas de 23) presta ayudas para las personas con diversidad funcional, frente al 22,92 \% de las universitarias (11 de 48 bibliotecas).

\section{Bibliotecas públicas estatales}

Como se ha comentado, ninguna carta de servicios de las bibliotecas públicas presta servicios para el colectivo multicultural y solo 8 de ellas $(34,78 \%)$ los ofrece para personas con diversidad funcional.

Por consiguiente, el análisis cualitativo de los servicios específicos se hará solo para este último colectivo. En este sentido, el lugar 'Dónde' se halla la información referente a este colectivo en las cartas de servicio de las 8 bibliotecas públicas, se ha englobado en un grupo común designado: 
"Servicios, compromisos e indicadores", es decir, el $100 \%$ de ellas pertenecen a una sola categoría. Y con respecto a 'Cómo' se indica dicha información, principalmente lo hacen: ofreciendo fondo bibliográfico con audiolibros, lenguaje de signos, etc., materiales especiales; u organizando actividades que involucran a personas con diversidad funcional.

\section{Bibliotecas universitarias}

En el caso de las bibliotecas universitarias con cartas de servicio cabe destacar que el porcentaje de servicios ofrecidos para ambos colectivos es bastante bajo en comparación con el número de cartas de servicio ya que, tal y como se ha comentado con anterioridad, de las 48 cartas de servicio sólo 2 hacen referencia al colectivo multicultural $(4,17 \%)$ y 11 a las personas con diversidad funcional $(22,92 \%)$.

En el análisis cualitativo de las bibliotecas universitarias cuyas cartas de servicio presentan ayudas para ambos grupos estudiados, en primer lugar, es importante destacar la escasez de bibliotecas universitarias involucradas con el colectivo multicultural, ya que muy pocas muestran en sus cartas de servicio ayudas para estas personas. Los 2 casos que hay $(100 \%)$ se localizan es en el apartado "Servicios" ('Dónde'). Y la forma en la que se presentan o 'Cómo' se reflejan es: mediante donaciones de material bibliográfico a cualquier usuario externo independientemente de su procedencia; o haciendo actividades dirigidas a la sociedad, tales como clubs de lectura, talleres y exposiciones de integración.

En segundo lugar, para el colectivo con diversidad funcional, 'Dónde' se localizan los servicios o recursos de las 11 bibliotecas que sí tienen este tipo de información es en la categoría "Servicios" (36,36 \%, 4 bibliotecas), "Compromisos" (36,36 $\%, 4$ bibliotecas) y "Otros: medidas, atención personalizada" (27,27 \%, 3 bibliotecas). Sobre 'Cómo' muestran estos servicios lo hacen: ofreciendo a las personas con diversidad funcional espacios, equipos y servicios adaptados a sus necesidades; o prestando servicios de atención personalizada a usuarios con limitación para el acceso a los recursos y servicios.

\subsection{Análisis de sedes web}

\subsubsection{Análisis general}

Se ha comprobado que tanto las 53 bibliotecas públicas del Estado como las 76 bibliotecas universitarias disponen de sede web, es decir, el $100 \%$ de ambas modalidades están representadas en la Web. Aunque era un hecho esperable, hemos querido comprobarlo, así como el buen funcionamiento de todas ellas. En ambos casos, el $100 \%$ estaban operativas en la fecha del análisis.

\subsubsection{Análisis de la multiculturalidad y la diversi- dad funcional en las sedes web}

Este análisis pretende comprobar si en las sedes web de las bibliotecas se hace referencia (principalmente, mediante servicios o recursos específicos) a la multiculturalidad y a las personas con diversidad funcional. El escrutinio de las sedes web para localizar dicha información ha sido realizado exclusivamente por los analistas.

Así, de las 53 bibliotecas públicas del estado, 15 $(28,30 \%)$ dispone de servicios para el colectivo multicultural y $23(43,40 \%)$ presentan ayudas para personas con diversidad funcional en sus sedes web. Si lo comparamos con las 76 bibliotecas universitarias, existe una diferencia significativa en el colectivo multicultural, ya que sólo 6 de ellas $(7,89 \%)$ cuenta con apoyo para este grupo y $33(43,42 \%)$ dispone de servicios para la diversidad funcional. Lo que sí se observa es un crecimiento general de los servicios en ambos colectivos (multicultural y diversidad funcional) y en ambos tipos de bibliotecas si lo comparamos con lo presentado en sus cartas de servicio. Es decir, las sedes web de las bibliotecas presentan más servicios para colectivos vulnerables que sus cartas de servicio.

\section{Bibliotecas públicas estatales}

De las 53 bibliotecas el 28,30\% presta servicios al colectivo multicultural (15 bibliotecas) y el $43,40 \%$ para diversidad funcional, es decir, 23 bibliotecas.

Se ha comentado que 15 sedes webs de bibliotecas públicas $(28,30 \%)$ prestan servicios para el colectivo multicultural. Cualitativamente, 'Dónde' se hallan recogidos dichos servicios o recursos es en cuatro grupos generales o categorías: "Biblioteca accesible" (33,33 \%, 5 bibliotecas), "Biblioteca multicultural" (33,33 \%, 5 bibliotecas), "Actividades" (20\%, 3 bibliotecas) y "Cultura diversa" (13,33 \%, 2 bibliotecas). 'Cómo' se refleja dicha información es: dotando a los municipios con población inmigrante de fondos en sus propias lenguas de origen; realizando actividades con las que pretenden hacer de la biblioteca un lugar de encuentro e intercambio cultural, o creando espacios multiculturales.

Con respecto a la diversidad funcional, el 43,40 $\%$ de las sedes web de las bibliotecas públicas del Estado (23 bibliotecas) tienen servicios o recursos para este colectivo. Se encuentran en tres apartados generales 'Dónde' se agrupan: "Biblioteca accesible" (78,26\%, 18 bibliotecas), "Servicios a medidas" (13,04\%, 3 bibliotecas) y "Actividades" (8,70 \%, 2 bibliotecas). Con respecto a 
'Cómo' se reflejan dichos servicios o recursos, la mayoría de las bibliotecas comprometidas, presentan colecciones dirigidas a personas que tienen dificultades lectoras permanentes (trastornos del aprendizaje, diversidad funcional, senilidad, etc.) ofreciendo letra grande, audiolibros, lectura fácil o lengua de signos; hacen talleres sobre dislexia y otras dificultades de aprendizaje o sobre personas con TEA (Trastorno del Espectro Autista); ofrecen servicio de préstamo a domicilio para personas mayores o con una discapacidad igual o superior al $33 \%$; ofrecen servicios para personas con dificultades auditivas (como películas con subtítulos), personas con dificultades visuales (como colección de materiales especiales, libros en Braille, en letra grande, audiolibros, telelupas, lupas de mano o atriles) o para personas con discapacidad intelectual (como libros de lectura fácil).

\section{Bibliotecas universitarias}

En las bibliotecas universitarias con sede web $(100 \%, 76)$ se observa una diferencia significativa entre los dos colectivos analizados, puesto que el número de servicios prestados es bastante más bajo para el colectivo multicultural con sólo un $7,89 \%$ (6 bibliotecas) que para las personas con diversidad funcional, con un 43,42 \% (33 bibliotecas).

En el análisis cualitativo de las 6 webs con servicios o recursos multiculturales $(7,89 \%)$, se han establecido tres categorías que agrupan los comentarios similares sobre 'Dónde' se encuentran dichos servicios. En "Orientación al estudiante" se recogen 4 bibliotecas $(66,67 \%)$, en la categoría "Compromiso social" se 1 biblioteca $(16,67 \%)$ y, por último, bajo la categoría "Espacio Violeta", otra biblioteca (16,67 \%). Sobre 'Cómo' se refleja esta información: mostrando una educación inclusiva y de atención a la diversidad, ofreciendo apoyos para que todos los estudiantes, a pesar de sus condiciones y situaciones específicas, tengan éxito en la Universidad y se vean suficientemente formados y capacitados para encontrar también su éxito profesional; participando en campañas de formación y sensibilización y cesión de espacios a ONG y otras entidades o asociaciones relacionadas con colectivos multiculturales; o creando un fondo especializado en estudios de género, focalizado en las dificultades de las mujeres, independientemente de su cultura y procedencia.

En cuanto a las 33 webs con servicios o recursos para el colectivo con diversidad funcional, 'Dónde' se han englobado es en las categorías: "Servicios accesibles/Espacios y equipos adaptados" (90,91 \%, 30 bibliotecas) y "Compromiso social" (9,09\%, 3 bibliotecas). En relación a 'Cómo' prestan servicios a las personas con diversidad funcional para facilitarles el uso y el acceso a sus recursos, encontramos: servicios focalizados mayoritariamente en la atención personalizada, poniendo en funcionamiento un conjunto de servicios para personas con discapacidad que les faciliten el acceso a la información como: servicio de sala, de préstamo, de información bibliográfica especializada, cursos de formación, puestos informáticos adaptados a sus necesidades $\mathrm{o}$ aseos adaptados.

\section{Conclusiones}

No se ha localizado ningún trabajo anterior que analice conjunta y comparativamente si en las cartas de servicio y en las sedes web de las bibliotecas públicas estatales y universitarias de España se exponen servicios y/o recursos para colectivos multiculturales y con diversidad funcional, por lo que la presente investigación puede representar un avance en una temática tan importante para la sociedad, en general, y las bibliotecas, en particular.

Con respecto a las cartas de servicio, tras un tedioso proceso de localización en las webs de las bibliotecas usando varios métodos para ello, disponen de ella solo la mitad aproximada, tanto de las bibliotecas públicas del Estado, como de las universitarias. Este resultado parece desfavorable, ya que no todas las bibliotecas públicas estatales y universitarias de España ponen a disposición del ciudadano sus cartas de servicio (herramienta de difusión, compromiso y calidad con los ciudadanos).

Con respecto a la ubicación de la carta de servicios, en ambos tipos de bibliotecas, se han identificado cinco apartados generales: "Biblioteca", "Conócenos", "Información", "Normativa" y "Servicios", que se encuentran, en su mayoría, en la página principal de las sedes web de las mismas, por ello, se puede concluir favorablemente que las bibliotecas con cartas de servicio las tienen accesibles en sus webs.

Tanto el colectivo multicultural, como las personas con diversidad funcional, se encuentran muy poco representados en las cartas de servicio de ambos tipos de bibliotecas, especialmente el colectivo multicultural, con porcentaje nulo en bibliotecas públicas y muy bajo en universitarias. El porcentaje en el colectivo con diversidad funcional asciende algo más, pero sigue siendo bajo, tanto en las bibliotecas públicas del Estado, como en las universitarias. La conclusión que se extrae de estos resultados es desfavorable, puesto que en la gran mayoría de las bibliotecas no se ha encontrado una referencia hacia uno de estos colectivos, y más aún cuando todas las bibliotecas tienen la misión común de garantizar el acceso a la 
información a todos los ciudadanos de forma gratuita y en igualdad de condiciones, independientemente de sus condiciones, posibilitando la formación continua y la integración social.

Sobre "Dónde" y "Cómo" se recogen los servicios y/o recursos para estos colectivos dentro de las cartas de servicio, en ambos tipos de bibliotecas y para ambos grupos, se han identificado dos secciones comunes "Servicios" y "Compromisos", donde se recopilan servicios y recursos destinados al colectivo multicultural como, por ejemplo, clubs de lectura, talleres y exposiciones de integración; y destinados al colectivo con diversidad funcional como, por ejemplo, fondo bibliográfico con audiolibros, lenguaje de signos, materiales especiales, etc. o servicios de atención personalizada para usuarios con limitaciones para el acceso a los recursos y servicios.

En relación a las sedes webs, a diferencia de las cartas de servicio, todas las bibliotecas públicas del Estado, así como las bibliotecas universitarias de España disponen de sede web y, además, todas están operativas, lo que representa un aspecto favorable. Además, su localización ha resultado sencilla utilizando las fuentes oficiales disponibles, aspecto que también resulta positivo.

Con respecto a la representación del colectivo multicultural y con diversidad funcional en las sedes web, el porcentaje para el colectivo multicultural sube algo en bibliotecas públicas y muy poco en universitarias, y para las personas con diversidad funcional, tanto en públicas como en universitarias, asciende y se sitúa cerca de la mitad de las mismas. Si bien estos resultados resultan, en todos los casos, más elevados que los obtenidos en las cartas de servicio, sobre todo en el caso de la diversidad funcional, podemos considerarlos desfavorables, ya que es importante fomentar, desde todas las sedes web de las bibliotecas, la sensibilización social acerca de los valores positivos de la multiculturalidad y la diversidad funcional.

Sobre "Dónde" y "Cómo" se recogen los servicios y/o recursos para estos colectivos dentro de las sedes web, las bibliotecas públicas recopilan bajo las secciones comunes "Biblioteca accesible" y "Actividades", los servicios y/o recursos para el colectivo multicultural, haciendo de la biblioteca un lugar de encuentro e intercambio cultural; y para el colectivo con diversidad funcional, recogiendo, por ejemplo, colecciones dirigidas a personas que tienen dificultades lectoras permanentes (ofreciendo letra grande, audiolibros, lectura fácil, lengua de signos, etc.). Las bibliotecas universitarias, por su parte, recogen bajo la sección principal "Orientación al estudiante" actividades enfocadas a los usuarios multiculturales como, por ejemplo, campañas de formación y sensibilización o cesión de espacios a ONG y a otras entidades relacionadas con este colectivo; y para las personas con diversidad funcional, en la sección principal "Servicios accesibles/Espacios y equipos adaptados" recopilan información y recursos como, por ejemplo, puestos informáticos adaptados.

\section{Agradecimientos}

Agradecemos la colaboración de los responsables de las bibliotecas que han atendido nuestras consultas.

\section{Notas}

(1) Bibliotecas Públicas del Estado (España): http://directoriobibliotecas. $\mathrm{mcu} . \mathrm{es} / \mathrm{dimbe} . \mathrm{cmd}$ ?apartado=buscador\& accion $=$ enviarAvanzado \& codigotipo $=30 \&$ codigotipo $2=31$ \&codigotitularidad=1

(2) Bibliotecas Universitarias (España): https://www.crue.org/universidades/

\section{Referencias}

Abraham, D. V. H. (1986). Two public libraries in India: Connemara and Kottayam. // Public Library Quarterly. 7:3/4, 49-60. doi: 10.1300/J118v07n03_07 (15/12/2019).

Aguillo, I. F (1998). Hacia un concepto documental de sede web. // El profesional de la información. http://www.elprofesionaldelainformacion.com/contenidos/1998/enero/hacia un concepto documental de sede web.html (01/03/2020)

Bushman, B.; Fagan, M.K. (2019). Public library programs and accommodations for deaf and hard-of-hearing children in the united states. // Journal of Deaf Studies and Deaf Education. 24:2, 74-83. doi:10.1093/deafed/eny041 $(10 / 01 / 2020)$

Candia, C.; Maderni, G. (2011), Las cartas de servicio como herramienta de mejora de la gestión pública. // INNOTEC Gestión. https://ojs.latu.org.uy/index.php/INNOTEC-Gestion/article/view/122 (22/11/2019)

Cervantes-Martínez, L.; Navas-Luque, M. (2013). Las bibliotecas públicas como instrumento para la integración social: un estudio de caso. // Migraciones. 34, 177-203. https://revistas.comillas.edu/index.php/revistamigraciones/article/view/2250/1976 (15/09/2020).

Cervantes-Martínez, L.; Navas-Luque, M.; Cuadrado-Guirado, I. (2015). Análisis de la prestación de servicios a inmigrantes en la red bibliotecas de Barcelona. // Revista Española de Documentación Científica. 38:3. doi:10. 3989/redc.2015.3.1192 (15/09/2020).

Cervantes-Martínez, L.; Navas-Luque, M.S.; Cuadrado-Guirado, I. (2019). Contacto intergrupal y actitudes en bibliotecas públicas: un estudio con usuarios marroquíes y españoles en Barcelona y Almería. // Revista Española de Documentación Científica. 42:1. https://doi.org/10.3989/ redc.2019.1.1581 (15/09/2020).

Everett, S. (2018). Visualizing the silent dialogue about race: Diversity outreach in an academic library. // Journal of Academic Librarianship. 44:4, 518-526. doi: 10.1016/j. acalib.2018.04.002 (14/11/2020)

García, F.; Monje, T. (2002). Biblioteca pública y servicios para la población inmigrante en las recomendaciones bibliotecarias internacionales. // España: Ministerio de Cultura, Subdirección General de Publicaciones, Información y Documentación. http://travesia.mcu.es/portalnb/jspui/ handle/10421/1176 (15/09/2019). 
González, L. (2014). ¿Qué es una carta de servicio? // bibliopos.es. https://www.bibliopos.es/que-es-una-carta-deservicios/ (Consulta: 01/03/2020).

Gómez-Hernández, J. A.; Hernández-Pedreño, M.; RomeroSánchez, E. (2017). Social and digital empowerment of vulnerable library users of the Murcia Regional Library, Spain. // El profesional de la información. 26:1, 20-32. http://eprints.rclis.org/30743/1/EPI_2017_020033_Gomez_Hernandez_Romero_Castellano.pdf (15/09/2019).

Green, R. A.; Huprich, J. (2009). Web accessibility and accessibility instruction. // Journal of Access Services. 6:1/2, 116136. doi: 10.1080/15367960802247825 (01/09/2019).

Horrigan, J. B. (2015). Libraries at the crossroads: The public is interested in new services and thinks libraries are important to communities. Washington: Pew Research Center. https://www.pewresearch.org/internet/2015/09/ 15/libraries-at-the-crossroads/ (15/09/2019).

IFLA (2001). Directrices IFLA/UNESCO para el desarrollo del servicio de bibliotecas públicas. // Madrid: Federación Internacional de Asociaciones de Bibliotecarios y Bibliotecas. https://www.ifla.org/files/assets/hq/publications/archive/the-public-library-service/pg01-s.pdf (20/02/2020).

IFLA (2008). Manifiesto IFLA por la biblioteca multicultural. https://archive.ifla.org/VII/s32/pub/MulticulturalLibraryManifesto-es.pdf (20/02/2020).

IFLA (2009). Comunidades multiculturales: directrices para el servicio bibliotecario. https://www.ifla.org/files/assets/library-services-to-multicultural-populations/publications/ multicultural-communities-es.pdf (20/02/2020).

Khailova, L. (2006). South carolinian public libraries' ADA accommodations to persons with disabilities. // Public Library Quarterly. 24:2, 19-46. doi: 10.1300/J118 v24n02_02 (10/11/2019).

Kumar, S. L.; Suresh, R. S. (2001). Strategies for providing effective reference services for international adult learners. // Reference Librarian. 33:69/70, 327-336. doi: 10.1300/J120v33n69_29 (15/02/2020).

Kumasi, K. D.; Manlove, N. L. (2015). Finding "diversity levers" in the core library and information science curriculum: A social justice imperative. // Library Trends. 64:2, 415-443. doi:10.1353/lib.2015.0047 (10/10/2019).

Lush, B. (2015). Managing accessible library web content. // Accessibility for Persons with Disabilities and the Inclusive Future of Libraries (Advances in Librarianship, 40). doi: 10. 1108/S0065-283020150000040017 (01/09/2019).

Magán, J. A; Gimeno, J. (2008). ¿Es "biblioteconómicamente correcto" el compromiso social de la biblioteca universitaria? El compromiso social en el trabajo bibliotecario. // Educación y Biblioteca. 166, 95-101. https://gredos.usal.es/bitstream/handle/10366/119558/EB20 N166 P95101. pdf;jsessionid=F7BA0EB4C15F19E62230F88 A6711D248?sequence=1 (15/02/2020)

Majinge, R. M.; Mutula, S. M. (2018). Access to electronic and print information resources by people with visual impairments in university libraries: A review of related literature. // Library Management. 39:6/7, 462-473. doi: 10.1108/LM04-2017-0038 (10/10/2019).

Millán Reyes, A. N. (2010). Servicios bibliotecarios: la perspectiva de los estudiantes con discapacidad y el personal bibliotecario a través de seis universidades madrileñas. // Revista Española de Documentación Científica. 33:1, 106-126. doi:10.3989/redc.2010.1.714 (15/02/2020).

Moral, C. del; Pedreira, G.; Sanz, J. R. (2008). Plan de accesibilidad en la red de Bibliotecas Públicas del Ayuntamiento de Madrid. http://travesia.mcu.es/portalnb/jspui/ bitstream/10421/553/1/com_367.pdf (16/05/2020).

Mulliken, A.; Djenno, M. (2017). Faculty visions for teaching web acc18/05/2020essibility within LIS curricula in the 1united states: A qualitative study. // Library Quarterly. 87:1, 36-54. doi: 10.1086/689313 (15/02/2020).

Mutula, S.; Majinge, R. M. (2016). Information behaviour of students living with visual impairments in university libraries: A review of related literature. // Journal of Academic Librarianship. 42:5, 522-528. doi: 10.1016/j.acalib.2016.06.019 (16/05/2020).

Novotny, E. (2003). Library services to immigrants: The debate in the library literature, 1900-1920, and a Chicago case study. // Reference and User Services Quarterly. 42:4, 342-352.

Oǧuz, E. S.; Kurbanoğlu, S. (2013). Strengthening social inclusion in multicultural societies through information literacy. // Bilgi Dunyasi. 14:2, 270-290.

Rodríguez Díaz, S.; Ferreira, M.A.V. (2010). Desde la dis-capacidad hacia la diversidad funcional. Un ejercicio de disnormalización. // Revista Internacional de Sociología. 68:2, 289-309. http://revintsociologia.revistas.csic.es/index.php/revintsociologia/article/view/333 (10/02/2020).

Sánchez-García, S.; Yubero, S. (2016). Papel socioeducativo de las bibliotecas públicas: nuevos perfiles profesionales para nuevos tiempos. // El profesional de la información. 25:2, 226-236. http://www.elprofesionaldelainformacion.com/contenidos/2016/mar/09.pdf (15/09/2019).

Santillán-Rivero, E.; Valles-Valenzuela, J. (2004). Las bibliotecas nacionales y los servicios para discapacitados. // Anales de documentación. 7, 1-13. https://revistas.um. es/analesdoc/article/view/1591/1641 (10/09/2020).

Schomberg, J.; Grace, M. (2005). Expanding a collection to reflect diverse user populations. // Collection Building. $24: 4, \quad 124-126$. doi: 10.1108/01604950510629282 (15/10/2019).

Serna Bilbao, M. de la. (2014). La regulación existente en la universidad española de los alumnos con discapacidad: Análisis de una experiencia concreta. https://ddd.uab.cat/pub/llibres/2014/126093/Congreso_ ACCEDES2014-p1134-1387.pdf (16/05/2020).

Small, R. V.; Myhill, W. N.; Herring-Harrington, L. (2015). Developing accessible libraries and inclusive librarians in the 21st century: Examples from practice. // Advanced in $\mathrm{Li}$ brarianship. 40, 73-88. doi: 10.1108/S0065-28302 0150000040013 (10/10/2020]

Thomas, R. L., Chiarelli-Helminiak, C.M., Ferraj, B. y Barrette, K. (2016). Building relationships and facilitating immigrant community integration: An evaluation of a cultural navigator program. // Evaluation and Program Planning. 55, 77-84. doi: 10.1016/j.evalprogplan.2015.11.003 (01/09/2020).

Varela-Prado, C.; Baiget, T. (2012). El futuro de las bibliotecas académicas: incertidumbres, oportunidades y retos. // Investigación bibliotecológica. 26:5, 115-135. http://w ww.revistas.unam.mx/index.php/ibi/article/view/33175 (15/09/2019).

Wang, H. (2012). Immigration in America: Library services and information resources. // Reference Services Review. 40:3, 480-511. doi: 10.1108/00907321211254715. $(15 / 09 / 2020)$.

\section{Apéndice 1}

\begin{tabular}{|c|c|c|c|}
\hline \multicolumn{4}{|c|}{ Bibliotecas públicas estatales con carta de servicios } \\
\hline Código & Denominación & Código & Denominación \\
\hline $\begin{array}{l}\text { BPE- } \\
\text { ALMERÍA }\end{array}$ & $\begin{array}{l}\text { Biblioteca } \\
\text { Pública del } \\
\text { Estado en } \\
\text { Almería - } \\
\text { Francisco } \\
\text { Villaespesa }\end{array}$ & BPE-LEÓN & $\begin{array}{l}\text { Biblioteca } \\
\text { Pública del } \\
\text { Estado en León }\end{array}$ \\
\hline
\end{tabular}




\begin{tabular}{|c|c|c|c|}
\hline $\begin{array}{l}\text { BPE- } \\
\text { ASTURIAS }\end{array}$ & $\begin{array}{l}\text { Biblioteca } \\
\text { Pública del } \\
\text { Estado en Gijón } \\
\text { - Jovellanos }\end{array}$ & $\begin{array}{l}\text { BPE- } \\
\text { MÁLAGA }\end{array}$ & $\begin{array}{l}\text { Biblioteca } \\
\text { Pública del } \\
\text { Estado en } \\
\text { Málaga }\end{array}$ \\
\hline BPE-ÁVILA & $\begin{array}{l}\text { Biblioteca } \\
\text { Pública del } \\
\text { Estado en Ávila }\end{array}$ & $\begin{array}{l}\text { BPE- } \\
\text { MELILLA }\end{array}$ & $\begin{array}{l}\text { Biblioteca } \\
\text { Pública del } \\
\text { Estado en } \\
\text { Melilla }\end{array}$ \\
\hline $\begin{array}{l}\text { BPE- } \\
\text { BADAJOZ }\end{array}$ & $\begin{array}{l}\text { Biblioteca } \\
\text { Pública del } \\
\text { Estado en } \\
\text { Badajoz - } \\
\text { Bartolomé J. } \\
\text { Gallardo }\end{array}$ & $\begin{array}{l}\text { BPE- } \\
\text { PALENCIA }\end{array}$ & $\begin{array}{l}\text { Biblioteca } \\
\text { Pública del } \\
\text { Estado en } \\
\text { Palencia }\end{array}$ \\
\hline $\begin{array}{l}\text { BPE- } \\
\text { BADAJOZ } 2\end{array}$ & $\begin{array}{l}\text { Biblioteca } \\
\text { Pública del } \\
\text { Estado en } \\
\text { Mérida - Jesús } \\
\text { Delgado } \\
\text { Valhondo }\end{array}$ & $\begin{array}{l}\text { BPE- } \\
\text { SALAMANCA }\end{array}$ & $\begin{array}{l}\text { Biblioteca } \\
\text { Pública del } \\
\text { Estado en } \\
\text { Salamanca - } \\
\text { Casa de las } \\
\text { Conchas }\end{array}$ \\
\hline $\begin{array}{l}\text { BPE- } \\
\text { CÁCERES }\end{array}$ & $\begin{array}{l}\text { Biblioteca } \\
\text { Pública del } \\
\text { Estado en } \\
\text { Cáceres - A. } \\
\text { Rodríguez- } \\
\text { Moñino/M. Brey }\end{array}$ & $\begin{array}{l}\text { BPE- } \\
\text { TENERIFE }\end{array}$ & $\begin{array}{l}\text { Biblioteca } \\
\text { Pública del } \\
\text { Estado en } \\
\text { Santa Cruz de } \\
\text { Tenerife }\end{array}$ \\
\hline BPE-CÁDIZ & $\begin{array}{l}\text { Biblioteca } \\
\text { Pública del } \\
\text { Estado en } \\
\text { Cádiz }\end{array}$ & $\begin{array}{l}\text { BPE- } \\
\text { SEVILLA }\end{array}$ & $\begin{array}{l}\text { Biblioteca } \\
\text { Pública del } \\
\text { Estado en } \\
\text { Sevilla - Infanta } \\
\text { Elena }\end{array}$ \\
\hline $\begin{array}{l}\text { BPE- } \\
\text { CANTABRIA }\end{array}$ & $\begin{array}{l}\text { Biblioteca } \\
\text { Central de } \\
\text { Cantabria / } \\
\text { Biblioteca } \\
\text { Pública del } \\
\text { Estado en } \\
\text { Santander }\end{array}$ & BPE-SORIA & $\begin{array}{l}\text { Biblioteca } \\
\text { Pública del } \\
\text { Estado en Soria }\end{array}$ \\
\hline $\begin{array}{l}\text { BPE- } \\
\text { CÓRDOBA }\end{array}$ & $\begin{array}{l}\text { Biblioteca } \\
\text { Pública del } \\
\text { Estado en } \\
\text { Córdoba }\end{array}$ & $\begin{array}{l}\text { BPE- } \\
\text { TOLEDO }\end{array}$ & $\begin{array}{l}\text { Biblioteca de } \\
\text { Castilla-La } \\
\text { Mancha / } \\
\text { Biblioteca } \\
\text { Pública del } \\
\text { Estado en } \\
\text { Toledo }\end{array}$ \\
\hline $\begin{array}{l}\text { BPE- } \\
\text { HUELVA }\end{array}$ & $\begin{array}{l}\text { Biblioteca } \\
\text { Pública del } \\
\text { Estado en } \\
\text { Huelva }\end{array}$ & $\begin{array}{l}\text { BPE- } \\
\text { VALLADOLID }\end{array}$ & $\begin{array}{l}\text { Biblioteca de } \\
\text { Castilla y León } \\
\text { Biblioteca } \\
\text { Pública del } \\
\text { Estado en } \\
\text { Valladolid }\end{array}$ \\
\hline BPE-JAÉN & $\begin{array}{l}\text { Biblioteca } \\
\text { Pública del } \\
\text { Estado en Jaén }\end{array}$ & $\begin{array}{l}\text { BPE- } \\
\text { ZAMORA }\end{array}$ & $\begin{array}{l}\text { Biblioteca } \\
\text { Pública del } \\
\text { Estado en } \\
\text { Zamora }\end{array}$ \\
\hline $\begin{array}{l}\text { BPE-LAS } \\
\text { PALMAS }\end{array}$ & $\begin{array}{l}\text { Biblioteca } \\
\text { Pública del } \\
\text { Estado en Las } \\
\text { Palmas de Gran } \\
\text { Canaria }\end{array}$ & & \\
\hline
\end{tabular}

\begin{tabular}{|c|c|c|c|}
\hline BU-ALI & $\begin{array}{l}\text { Universidad de } \\
\text { Alicante }\end{array}$ & BU-LRIO & $\begin{array}{l}\text { Universidad de La } \\
\text { Rioja }\end{array}$ \\
\hline $\begin{array}{l}\text { BU- } \\
\text { AMAD }\end{array}$ & $\begin{array}{l}\text { Universidad de } \\
\text { Alcalá }\end{array}$ & BU-MA & $\begin{array}{l}\text { Biblioteca de la } \\
\text { Universidad de } \\
\text { Málaga - Biblioteca } \\
\text { General }\end{array}$ \\
\hline BU-BAD & $\begin{array}{l}\text { Universidad de } \\
\text { Extremadura }\end{array}$ & BU-MALL & $\begin{array}{l}\text { Universitat de les } \\
\text { Illes Balears }\end{array}$ \\
\hline BU-BAR & $\begin{array}{l}\text { Universitat de } \\
\text { Barcelona }\end{array}$ & $\begin{array}{l}\text { BU- } \\
\text { MHALI }\end{array}$ & $\begin{array}{l}\text { Universidad Miguel } \\
\text { Hernández de } \\
\text { Elche }\end{array}$ \\
\hline BU-BUR & $\begin{array}{l}\text { Biblioteca de la } \\
\text { Universidad de } \\
\text { Burgos }\end{array}$ & BU-MUR & $\begin{array}{l}\text { Universidad de } \\
\text { Murcia }\end{array}$ \\
\hline $\begin{array}{l}\text { BU- } \\
\text { C3MAD }\end{array}$ & $\begin{array}{l}\text { Universidad Carlos } \\
\text { III de Madrid }\end{array}$ & $\begin{array}{l}\text { BU- } \\
\text { NEDMAD }\end{array}$ & $\begin{array}{l}\text { Universidad } \\
\text { Nacional de } \\
\text { Educación a } \\
\text { Distancia }\end{array}$ \\
\hline $\mathrm{BU}-\mathrm{CA}$ & $\begin{array}{l}\text { Biblioteca de la } \\
\text { Universidad de } \\
\text { Cádiz - Servicio } \\
\text { Central de } \\
\text { Bibliotecas }\end{array}$ & BU-PAM & $\begin{array}{l}\text { Universidad Pública } \\
\text { de Navarra }\end{array}$ \\
\hline $\begin{array}{l}\text { BU- } \\
\text { CAMUR }\end{array}$ & $\begin{array}{l}\text { Universidad Católica } \\
\text { San Antonio de } \\
\text { Murcia }\end{array}$ & BU-PCAT & $\begin{array}{l}\text { Universitat } \\
\text { Politècnica de } \\
\text { Catalunya }\end{array}$ \\
\hline BU-CAN & $\begin{array}{l}\text { Biblioteca de la } \\
\text { Universidad de } \\
\text { Cantabria }\end{array}$ & $\begin{array}{l}\text { BU- } \\
\text { PCMAD }\end{array}$ & $\begin{array}{l}\text { Universidad } \\
\text { Pontificia Comillas }\end{array}$ \\
\hline BU-CAS & Universitat Jaume I & $\begin{array}{l}\text { BU- } \\
\text { PFBAR }\end{array}$ & $\begin{array}{l}\text { Universitat Pompeu } \\
\text { Fabra }\end{array}$ \\
\hline $\begin{array}{l}\text { BU- } \\
\text { CHVAL }\end{array}$ & $\begin{array}{l}\text { Universidad CEU } \\
\text { Cardenal Herrera }\end{array}$ & $\begin{array}{l}\text { BU- } \\
\text { PMAD }\end{array}$ & $\begin{array}{l}\text { Universidad } \\
\text { Politécnica de } \\
\text { Madrid }\end{array}$ \\
\hline BU-CLM & $\begin{array}{l}\text { Biblioteca de la } \\
\text { Universidad de } \\
\text { Castilla-La Mancha }\end{array}$ & $\begin{array}{l}\text { BU- } \\
\text { POSEV }\end{array}$ & $\begin{array}{l}\text { Biblioteca de la } \\
\text { Universidad Pablo } \\
\text { de Olavide }\end{array}$ \\
\hline $\begin{array}{l}\text { BU- } \\
\text { CMAD }\end{array}$ & $\begin{array}{l}\text { Universidad } \\
\text { Complutense de } \\
\text { Madrid }\end{array}$ & BU-PSAL & $\begin{array}{l}\text { Biblioteca de la } \\
\text { Universidad } \\
\text { Pontificia de } \\
\text { Salamanca }\end{array}$ \\
\hline $\mathrm{BU}-\mathrm{CO}$ & $\begin{array}{l}\text { Biblioteca de la } \\
\text { Universidad de } \\
\text { Córdoba - Biblioteca } \\
\text { General }\end{array}$ & BU-PVAS & $\begin{array}{l}\text { Euskal Herriko } \\
\text { Unibertsitatea }\end{array}$ \\
\hline $\begin{array}{l}\text { BU- } \\
\text { CVAL }\end{array}$ & $\begin{array}{l}\text { Universidad Católica } \\
\text { de Valencia San } \\
\text { Vicente Mártir }\end{array}$ & BU-SEV & $\begin{array}{l}\text { Biblioteca de la } \\
\text { Universidad de } \\
\text { Sevilla }\end{array}$ \\
\hline $\begin{array}{l}\text { BU- } \\
\text { EMAD }\end{array}$ & $\begin{array}{l}\text { Universidad } \\
\text { Europea de Madrid }\end{array}$ & BU-TARR & $\begin{array}{l}\text { Universitat Rovira i } \\
\text { Virgili }\end{array}$ \\
\hline $\begin{array}{l}\text { BU- } \\
\text { FVMAD }\end{array}$ & $\begin{array}{l}\text { Universidad } \\
\text { Francisco de Vitoria }\end{array}$ & BU-TEN & $\begin{array}{l}\text { Biblioteca de la } \\
\text { Universidad de La } \\
\text { Laguna }\end{array}$ \\
\hline BU-GR & $\begin{array}{l}\text { Biblioteca de la } \\
\text { Universidad de } \\
\text { Granada }\end{array}$ & BU-VAL & $\begin{array}{l}\text { Universitat de } \\
\text { València }\end{array}$ \\
\hline BU-HU & $\begin{array}{l}\text { Biblioteca de la } \\
\text { Universidad de } \\
\text { Huelva - Biblioteca } \\
\text { La Merced }\end{array}$ & BU-VALL & $\begin{array}{l}\text { Universidad de } \\
\text { Valladolid }\end{array}$ \\
\hline $\begin{array}{l}\text { BU- } \\
\text { JAEN }\end{array}$ & $\begin{array}{l}\text { Biblioteca de la } \\
\text { Universidad de Jaén }\end{array}$ & BU-VIG & $\begin{array}{l}\text { Universidade de } \\
\text { Vigo }\end{array}$ \\
\hline BU-LCO & $\begin{array}{l}\text { Biblioteca de la } \\
\text { Universidad Loyola } \\
\text { Andalucía }\end{array}$ & BU-ZAR & $\begin{array}{l}\text { Biblioteca de la } \\
\text { Universidad de } \\
\text { Zaragoza }\end{array}$ \\
\hline
\end{tabular}

\section{Apéndice 2}

\begin{tabular}{llll}
\hline \multicolumn{3}{l}{ Bibliotecas universitarias con carta de servicios } \\
\hline Código & Denominación & Código & Denominación \\
\hline $\begin{array}{l}\text { BU- } \\
\text { ABAR }\end{array}$ & $\begin{array}{l}\text { Universitat } \\
\text { Autònoma de } \\
\text { Barcelona }\end{array}$ & BU-LEÓ & $\begin{array}{l}\text { Biblioteca de la } \\
\text { Universidad de } \\
\text { León }\end{array}$ \\
\hline BU- & Universidade da & BU-LLEl & $\begin{array}{l}\text { Universitat de } \\
\text { Lleida }\end{array}$ \\
ACOR & Coruña & & $\begin{array}{l}\text { Biblioteca de la } \\
\text { Universidad de Las } \\
\text { Palmas de Gran } \\
\text { Canaria }\end{array}$ \\
\hline BU-AL & Biblioteca de la & BU-LPAL \\
& Universidad de & & \\
& Almería - Nicolás & &
\end{tabular}

Enviado: 2021-03-29. Segunda versión: 2021-06-04. Aceptado: 2021-06-17. 